\title{
Stress Reducing Effects of Indoor Plants in a Classroom Setting
}

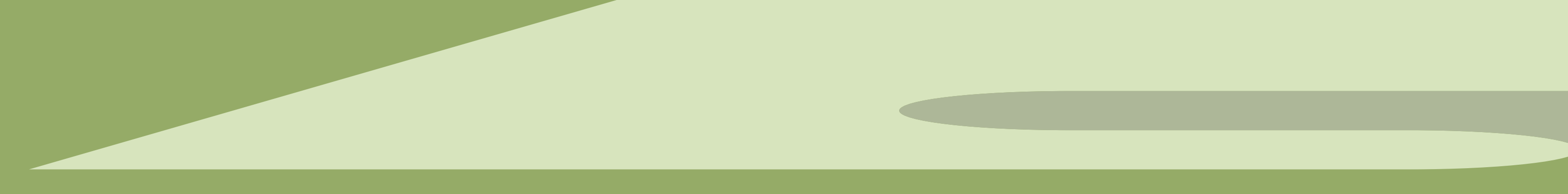

\author{
Laura Tennant
}

Dominican University of California

\section{Introduction}

Natural settings and access to plants appear to have significant beneficial effects on people (Relf, 1992).

Studies have shown that views of plants reduce stress, anger, and feelings of pain (Ulrich, 1984, Beukeboom et al., 2012, Park \& Mattson, 2008).

Beyond the labs, big businesses are designing natural elements into their buildings.

In the classroom, stress can impact

reasoning, reaction time, language, attention control, and memory.

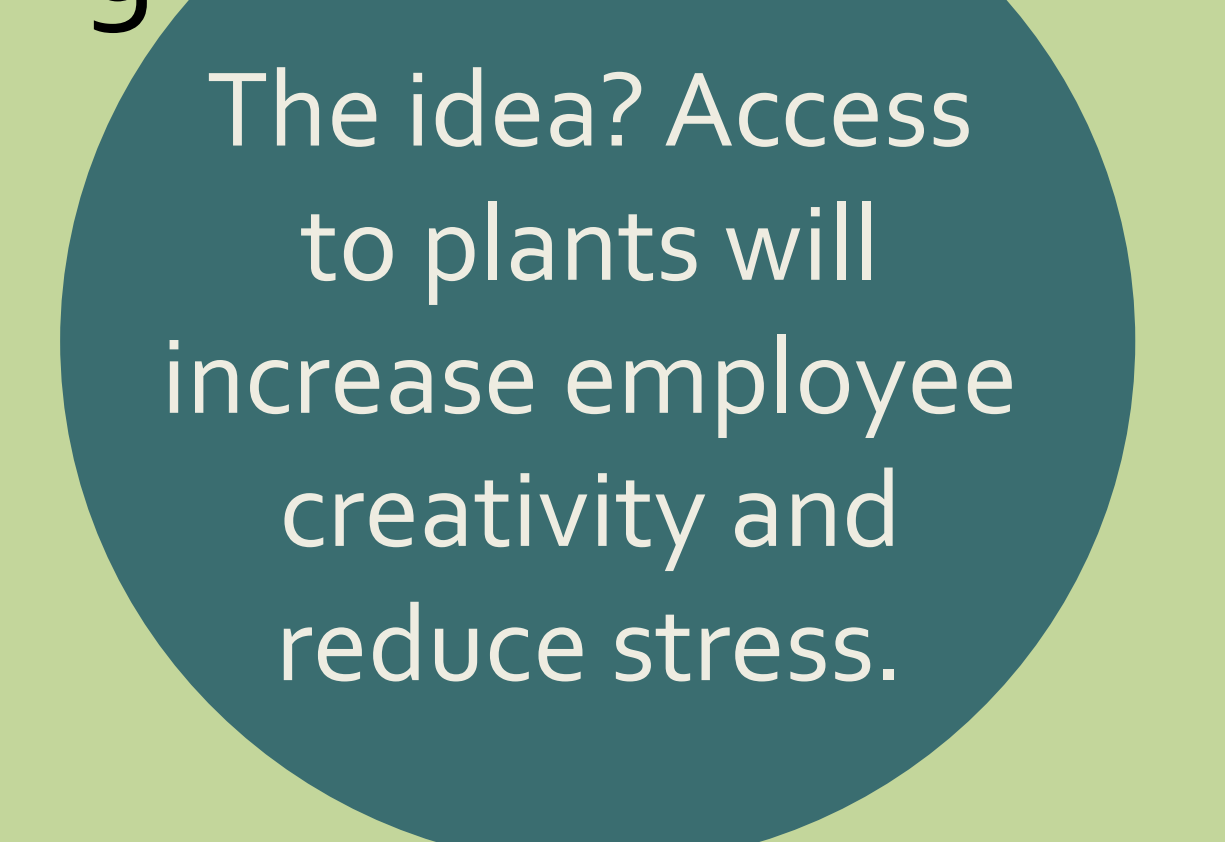

Recent studies have shown that natural views from a

classroom not only reduced student stress and fear, but also resulted in an improved ability to focus (Li \& Sullivan, 2016).

$$
\begin{array}{r}
\text { Hypotheses } \\
\hline
\end{array}
$$

The introduction of visible indoor plants to a classroom will result in:

- Reduced stress

- Reduced mental fatigue

- Improved performance on cognitive test

\section{Method}

An experimental design was used to study the effects of indoor plants on the reduction of stress and mental fatigue.

$\mathbf{4 8}$ college students and non-students were recruited from a liberal arts college in Marin County. $81 \%$ were female, the mean age was $32.88(S D=15.93)$

Participants were split into two groups - one in a classroom with indoor plants, and one in a classroom without plants. Both groups were given 10 minutes to complete identical 12-question math tests.

After the test, participants completed a 12-question survey. They rated their levels of stress and mental fatigue on a 4-point Likert scale ( $1=$ not at all; $4=$ very much)

\section{Measures}

Experienced stress was measured using six items from the State-Trait Anxiety Inventory, and three items from the Profile of Mood States. The scales are designed to measure anxiety.

- Anxiety absent: "I feel calm"

- Anxiety present: "I feel upset"

- Tension: "I feel anxious"

Experienced fatigue was measured using three items

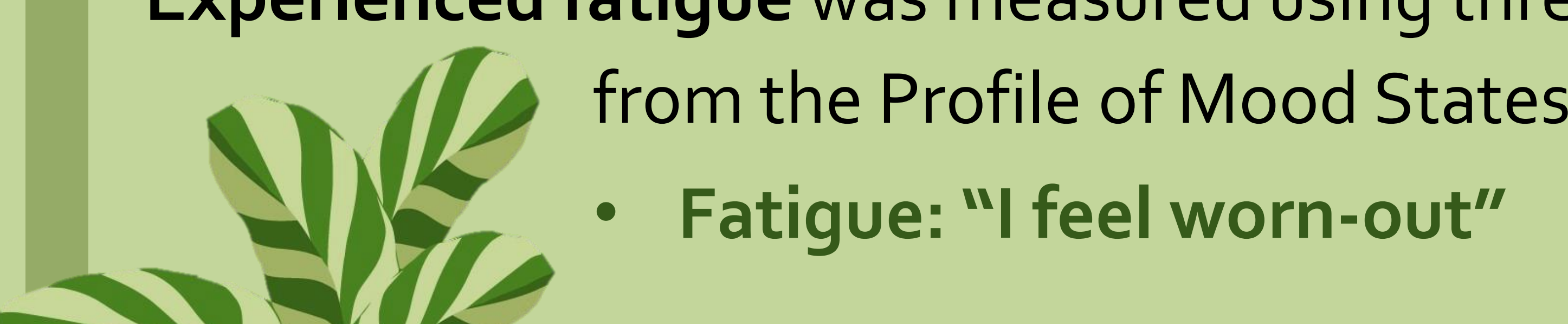

- Fatigue: "I feel worn-out"

Test performance was measured by the number of math questions answered correctly.

\section{Results}

Three independent samples t-tests demonstrated no significant differences between the two groups. Both the control and experiment groups experienced a similar level of anxiety $(t(45)=.176, p>.05)$, tension $(t(45)=$ $-.517, p>.05)$, and mental fatigue $(t(45)=-.020, p>.05)$.

A fourth independent samples t-test demonstrated no significant difference in the performance on the cognitive test between control and experiment groups $(t(46)=-.284, p>.05)$.

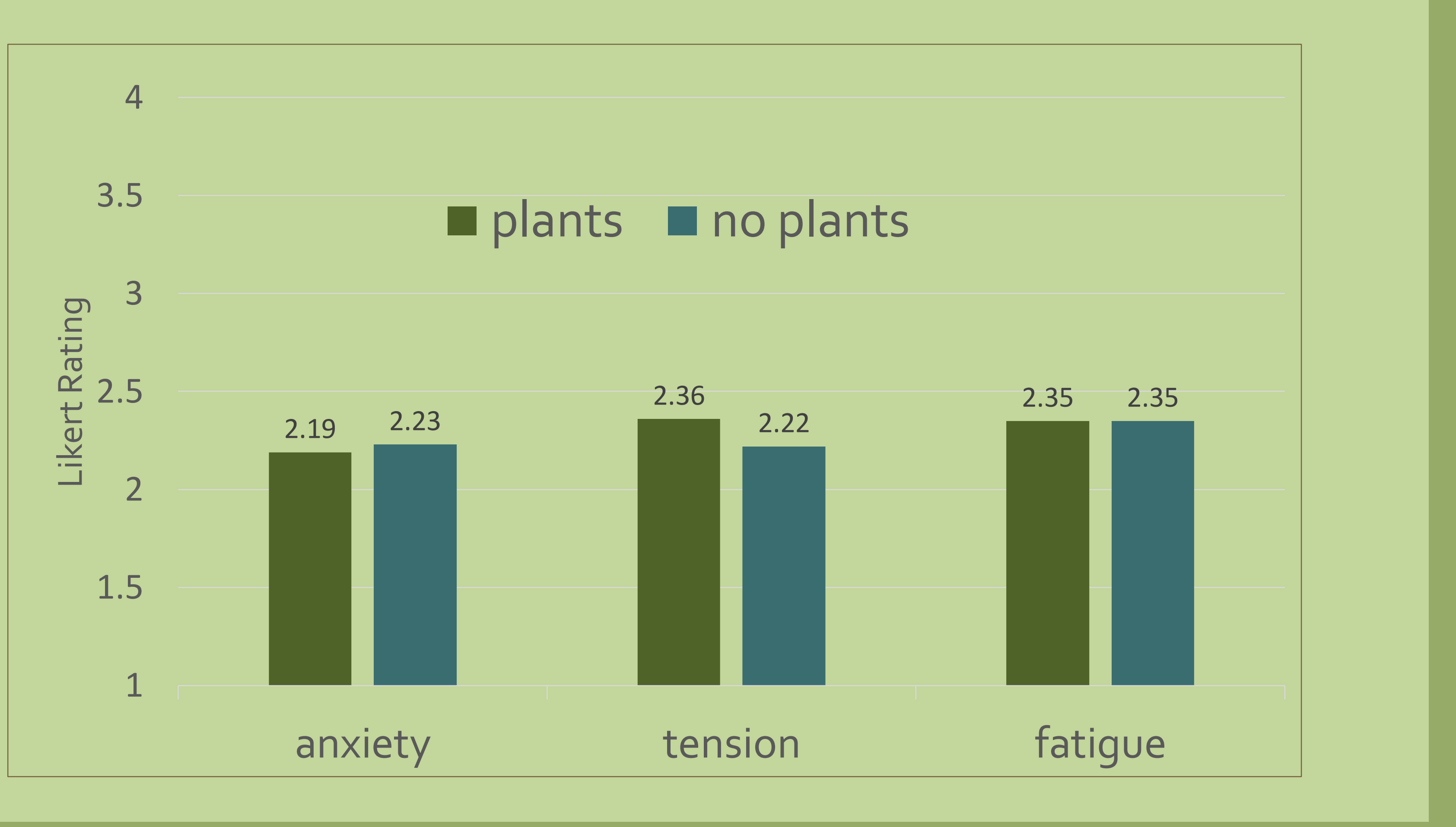

\section{Discussion}

There is a body of work dedicated to the study of the health benefits of plants for humans. The most recent studies of the past 30 years have demonstrated these benefits in a variety of settings.

The results of the current study do not support the hypotheses. This outcome was the result of limitations in this specific experiment, and not a confirmation that the hypotheses are false.

In studies that have shown stress relief in the presence of plants, study subjects had was not enough to have a significant impact.
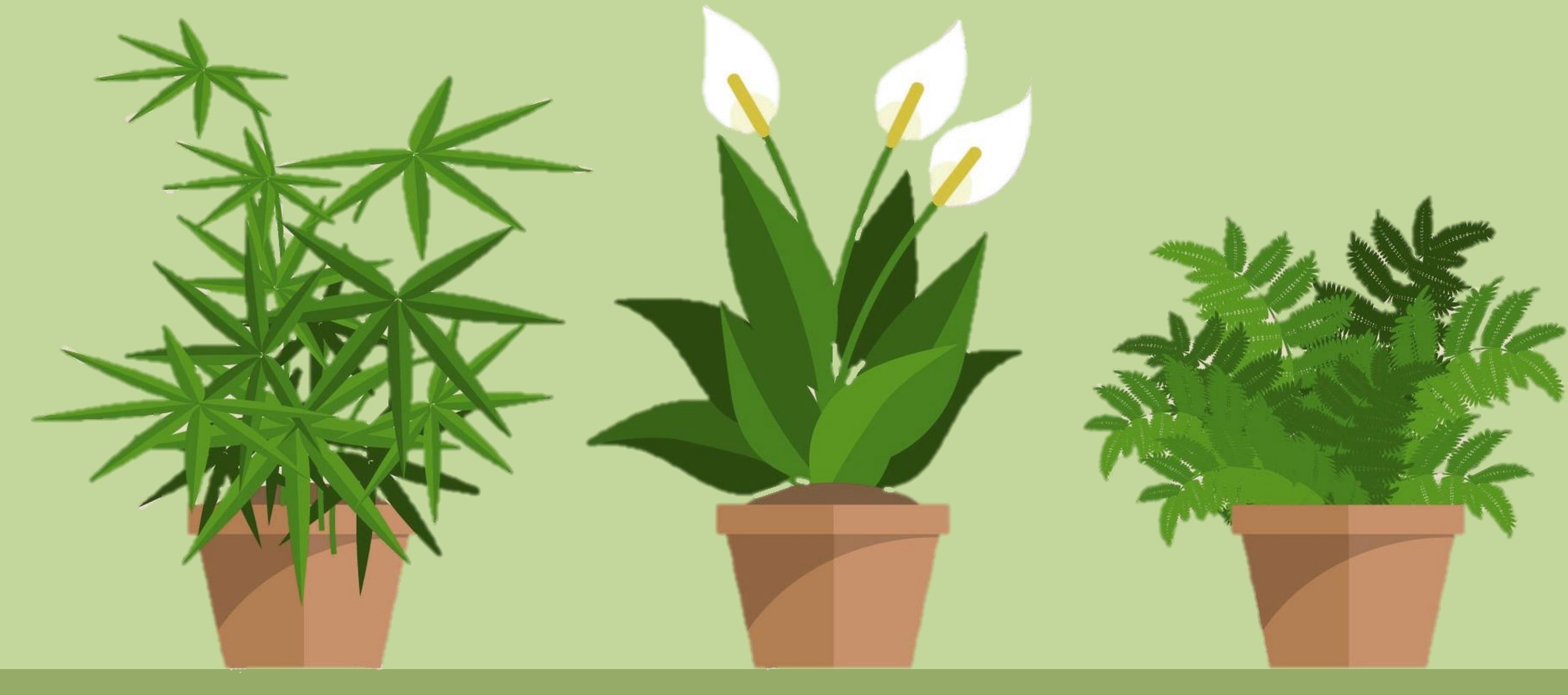

\section{Future Direction}

Most of the studies done on the health benefits of plants have taken place in Europe and North America. Future studies could look at the plant/human relationship in other countries and cultures.

What are some other advantages of exposure to plants and natural environments? Future studies could assess social and spiritual well-being.

Schools, medical centers, and businesses are incorporating natural elements as studies continue to demonstrate benefits. Stress reduction, improved focus and performance, and increased healing rates will become even more evident as the ability to study these effects becomes widespread. 